\title{
Premelting Dynamics: Geometry and Interactions
}

\author{
J. S. Wettlaufer, ${ }^{* \dagger, \S}$ M. G. Worster, ${ }^{\ddagger}$ and L. A. Wilen ${ }^{\S, \perp}$ \\ Applied Physics Laboratory, Box 355640, and Department of Physics, Box 351560, University of Washington, \\ Seattle, Washington 98195, and Institute of Theoretical Geophysics, Department of Applied Mathematics and \\ Theoretical Physics, University of Cambridge, Cambridge CB3 9EW, England
}

Received: October 11, $1996^{\otimes}$

\begin{abstract}
Recent advances in understanding the evolution of interfacially melted water films are discussed from several perspectives. The essential mechanism is the motion of thin films under the influence of thermomolecular pressure gradients. The mechanism is common to volatile liquid films at the interface between a solid and its vapor or a solid and an uncorrugated inert substrate, and the dynamics is theoretically similar to the wetting of an uncorrugated inert substrate by a nonvolatile liquid. New theoretical treatments of this process focus on the intermolecular origin of the thermomolecular pressure gradient, its time dependence, and the role of substrate geometry. Strikingly different results obtain by varying these three features. The experimental realization of these dynamics involves ice single crystals against a polymer interface.
\end{abstract}

In wetting phenomena microscopic interactions have observable macroscopic implications and thereby capture the interest of a broad range of researchers. In the most general description of wetting, classical surface thermodynamics arises from considerations of intermolecular potentials..$^{1,2}$ The critical behavior of solids, liquids, and gases is studied with sufficient generality that extensions to magnetic systems, for example, are immediate, and further extensions drive studies of complex fluids $^{3}$ and biomembranes. ${ }^{4}$ Our interest lies in bringing the phenomenology of wetting to bear on interfacial premelting, its general extension to driven systems, and specific realizations of these in ice. In so doing, we have been able to extract a fundamental mechanism of frost heave in natural and laboratory systems and provide some new insights into dynamical wetting phenomena distant from the system studied herein.

Surface melting is a wetting phenomenon at the interface between a solid and its vapor phase at temperatures below the bulk freezing point $T_{\mathrm{m}}$. If the interface is wet by the supercooled liquid phase, there must be a reduction in interfacial free energy to offset the increase in bulk free energy associated with the formation of the film. If we replace the vapor phase by a wall of a different material, the process is referred to as interfacial melting and the mean field thermodynamic description is essentially the same. The melting process may be complete or incomplete (e.g., refs 5 and 6). In the former case the film thickness diverges as $T_{\mathrm{m}}$ is approached from below, and in the latter case the film growth is truncated at finite undercooling commonly under the influence of retarded potential effects. The basic competition driving the process is that between adhesion and cohesion.

A primary motivation for understanding interfacial melting at ice surfaces concerns the important role it can play in a host of environmental problems ranging from the sintering of snowpacks to polar stratospheric cloud chemistry. ${ }^{5}$ Of particular importance in cold climates is the dramatic deformation of water saturated soils known generically as "frost heave". Although it is clearly not caused by the volume expansion of water during

\footnotetext{
Applied Physics Laboratory, University of Washington.

University of Cambridge.

$\S$ Department of Physics, University of Washington.

${ }^{\perp}$ Present address: Department of Physics and Astronomy, Ohio University, Athens, $\mathrm{OH} 45701$.

${ }^{\otimes}$ Abstract published in Advance ACS Abstracts, June 15, 1997.
}

solidification, we are just beginning to tease out the fundamental causes originating in premelting phenomena. The link between stable liquid water at subfeezing interfaces and frost heave was established many years ago, but the varied causes of this water have obscured attempts to extract the fundamental mechanisms driving frost heave. In a porous medium, curvature, confinement, interfacial roughness and disorder, impurities, and interfacial premelting all contribute to the finite volume fraction of water at subfreezing temperatures. Individually, these effects have distinct temperature dependencies, but their combined effects have thus far limited understanding of frost heave to semiempirical treatments. For this reason we have focused on isolating the role of interfacial melting in the existence and mobility of unfrozen interfacial water. The intermolecular origin of the films, the effect of external forcing, and the geometry of the confining wall are the main features of our studies. Theoretical developments are tested against recent experiments on isolated crystalline surfaces.

There are several approaches to the problem, varying from microscopic to macroscopic (e.g., refs 2 and 5-8), which focus on understanding thermodynamic equilibrium. Since we are ultimately interested in dynamical behavior, we begin by writing down the mean field grand potential $\Omega$ for the system which is the natural free energy for describing interphase pressure differences. $^{9}$ The total free energy for a layered wall (w), film (1), solid (s) system of area $A_{i}$ is written as a combination of bulk and surface terms:

$$
\Omega=-P_{1} V_{1}-P_{\mathrm{s}} V_{\mathrm{s}}+\mid(\mathrm{d})
$$

Here $P$ and $V$ denote pressure and volume and the liquid film has a thickness $d$. The interfacial term I $(d)=\left(\Delta \gamma f(d)+\tilde{\gamma}_{\mathrm{SW}}\right) A_{i}$ is a phenomenological representation of the potential between the three layers under the assumption that the volume-volume interactions are of a power law form. We write $f(d)=1-$ $(\sigma / d)^{\nu-1}$, where $\sigma$ is on the order of a molecular diameter and $v$ depends on the nature of the interactions. Representative values include $v=3(v=4)$ for nonretarded (retarded) van der Waals interactions and $v=2(v=3 / 2)$ for long-range (shortrange) electrostatic interactions. By electrostatic interfacial interactions we refer to a simple treatment in which the substrate possesses a surface charge density and the confinement of counterions present in the liquid creates a repulsive force across 
the layer. ${ }^{6}$ By definition, $\Delta \gamma=\gamma_{\mathrm{sl}}+\gamma_{\mathrm{lw}}-\tilde{\gamma}_{\mathrm{sw}}$, where the $\gamma^{\prime}$ 's are the solid-liquid (sl), liquid-wall (lw), and solid-wall (sw) interfacial free energies, but note that the short-range cutoff defined by the value of $\sigma$ is intimately wed to the effective nature of the solid-wall coefficient $\tilde{\gamma}_{\text {sw }}$ (see Appendix of ref 9). This form of $\mid(d)$ captures the essential features of more detailed treatments of complete interfacial melting under the influence of dispersion or electrostatic forces ${ }^{6}$ and allows us to develop a dynamical theory for arbitrary power law interactions and to make predictions relevant to experimental systems. The grand potential finds a minimum at fixed temperature and chemical potential, yielding two very useful results specific to the nature of the interactions. To first order in the reduced temperature $t_{\mathrm{r}}$ $=\left(T_{\mathrm{m}}-T\right) / T_{\mathrm{m}}$, the film thickness is

$$
d=\left(-\frac{(\nu-1) \sigma^{\nu-1} \Delta \gamma}{\rho_{\mathrm{s}} q_{\mathrm{m}}}\right)^{1 / v} t_{\mathrm{r}}^{-1 / v} \equiv \lambda_{\nu} t_{\mathrm{r}}^{-1 / v}
$$

where $\rho_{\mathrm{s}}$ is the density of the solid and $q_{\mathrm{m}}$ is the latent heat of fusion. The coefficient $\lambda_{v}$ depends on the interaction $v$; for example, when $v=3, \sigma^{2} \Delta \gamma=A / 12 \pi$, where $A$ is the Hamaker constant. The pressure in each phase is uniform, but the interfacial interactions create a pressure difference between the film and the bulk solid

$$
P_{1}-P_{\mathrm{s}}=\Delta \gamma(v-1) \sigma^{\nu-1} d^{-v}
$$

the solid repels the wall through the liquid, in analogy with the disjoining pressure of wetting. When taken together these results yield a thermodynamic statement that is independent of the nature of the interactions and helps to describe the main ingredient of premelting dynamics:

$$
P_{1}=P_{\mathrm{s}}-\rho_{\mathrm{s}} q_{\mathrm{m}} t_{\mathrm{r}}
$$

We imagine that the pressure in the solid is fixed by contact with a reservoir. Therefore, the pressure in the liquid film increases with temperature, and, by imposing a temperature gradient parallel to the interface, the resulting thermomolecular pressure gradient will drive premelted liquid from high to low temperature. The maximum pressure in the case of water is approximately $11 \mathrm{~atm}^{-1}$, so thermomolecular pressure driven flow as a frost heave mechanism captures our attention. These dynamics make mathematical contact with other wetting phenomena, such as when there is a gradient in the disjoining pressure along an inert wetting substrate or the competition between interfacial repulsion and gravity that Helium films exhibit when climbing over container walls. ${ }^{12}$ An essential distinction is that, in premelting, the film thickness is determined by the temperature, thinning as the temperature decreases, so that in a driven system continuity requires that liquid will convert to solid during its transit toward lower temperatures.

The detailed dynamics depends on the nature of the interactions responsible for the film, the thermomolecular pressure gradient, the liquid/solid conversion rate, and the geometry and mechanics of the confining wall. We call the pressure exerted by the confining wall the "overburden pressure" by analogy with the frost heave terminology, and if it takes a value greater than the maximum pressure (found by setting $P_{1}$ to the reservoir pressure in eq 4) in the film, then flow will cease, but if it is less than the maximum, flow will persist. The thermomolecular pressure gradient is written as

$$
\nabla P_{1}=-\rho_{1} q_{\mathrm{m}} \nabla t_{\mathrm{r}}+\frac{\rho_{\mathrm{l}}}{\rho_{\mathrm{s}}} \nabla P_{\mathrm{s}}
$$

where we treat $P_{\mathrm{s}}$ as the external pressure exerted on the solid by the confining wall/membrane..$^{9-11,13}$ For our studies the radius of curvature of the deformed membrane is always large compared with $d$, so the membrane exerts a pressure deep within the solid. We assume that any solid deformation is slow relative to the film dynamics. We have studied the configurations shown in Figures 1 and 3. The common features are that the film thickness depends solely on the temperature, relaxing to the value determined by eq 2 on a time scale negligible relative to that for heat conduction. The confining wall evolves in time according to the spatial variation in the thin film volume flux $Q \propto-d^{3} \nabla P_{1}$, with the proportionality constant depending on the geometry. ${ }^{9,11}$ Finally, if the imposed temperature gradient is transient, a heat conservation equation must be included. ${ }^{9}$

In Figure 1 we display frost heave in a flexible capillary tube, a geometry chosen because of its broad applicability to both physical and biological systems. For this example we focus on nonretarded van der Waals interactions $(v=3)$. We imagine initiating an experiment by cooling the cold ring to a value $T_{\mathrm{f}}$ $<T_{\mathrm{m}}$ so that the vertical solid-liquid interface at $x_{\mathrm{m}}(t)$ grows axially along the capillary. The temperature $T(x, t)$ in the region $0<x<x_{\mathrm{m}}$ drives an axisymmetric thermomolecular pressure gradient. The film thickness $d(x, t)=r_{2}(x, t)-r_{1}(x, t)$ depends on position through the temperature. The volume flux through the annular premelted region is then

$$
\mathrm{Q}=\frac{r_{2} d^{3}}{6 \eta}\left(\frac{\rho_{1}}{\rho_{\mathrm{s}}} \partial_{x} P_{\mathrm{s}}-\rho_{1} q_{\mathrm{m}} \partial_{x} \frac{\left(T_{\mathrm{m}}-T\right)}{T_{\mathrm{m}}}\right)
$$

where $\eta$ is the dynamic viscosity of the bulk supercooled liquid. Hence, continuity demands that any change in the radius of the tube is in response to variations in the volume flux

$$
\partial_{t} \pi r_{2}^{2}+\partial x \mathrm{Q}=0
$$

and $t$ denotes time. The pressure at each point $x$ in the solid is opposed by a restoring hoop stress exerted by the capillary membrane, and we assume linear elasticity, $P_{\mathrm{s}}=k\left(r_{2}-r_{0}\right)$, where $k$ is a constant and $r_{0}$ is the undeformed radius of the capillary tube. Combining the last two expressions gives an evolution equation for the capillary radius $r_{2}$ and the temperature $T$ as

$$
\partial_{t} r_{2}-\frac{\rho_{1} \pi k \lambda^{3}}{12 \eta \rho_{\mathrm{s}}} \partial_{x}\left[\frac{T_{\mathrm{m}}}{T_{\mathrm{m}}-T} \partial_{x}\left\{r_{2}-r_{0}-\frac{\rho_{\mathrm{s}} q_{\mathrm{m}}}{\pi k T_{\mathrm{m}}}\left(T_{\mathrm{m}}-T\right)\right\}\right]=0
$$

The temperature gradient along the solid drives solidification of ice down the tube, and latent heat is released as liquid in the thin film solidifies. Hence, heat conservation gives us the other required equation

$$
\partial_{t} T=\kappa \partial_{x x}^{2} T+\frac{2 q_{\mathrm{m}}}{c_{p} r_{0}} \partial_{t} r_{2}
$$

where $c_{p}$ and $\kappa$ are the specific heat at constant pressure and the thermal diffusivity of the solid, which will carry almost all of the heat. ${ }^{9}$ This completes the set of evolution equations which admit a similarity solution and are then solved analytically, subject to four boundary conditions and an auxiliary condition, using the method of matched asymptotic expansions. ${ }^{9}$ The main result, for the dimensionless radius $R(\xi)=\left(r_{2}-\right.$ $\left.r_{0}\right) \beta^{-1}$, is shown in Figure 2a. The length scale characterizing the deformation of the wall $\beta=\rho_{\mathrm{s}} q_{\mathrm{m}} \Delta T / \pi k T_{\mathrm{m}}$ is the product of the thermomolecular pressure coefficient $\rho_{\mathrm{s}} \mathrm{q}_{\mathrm{m}} / T_{\mathrm{m}}$ and the ratio of the thermal drive $\Delta T=T_{\mathrm{m}}-T_{\mathrm{f}}$ to the strength of the 

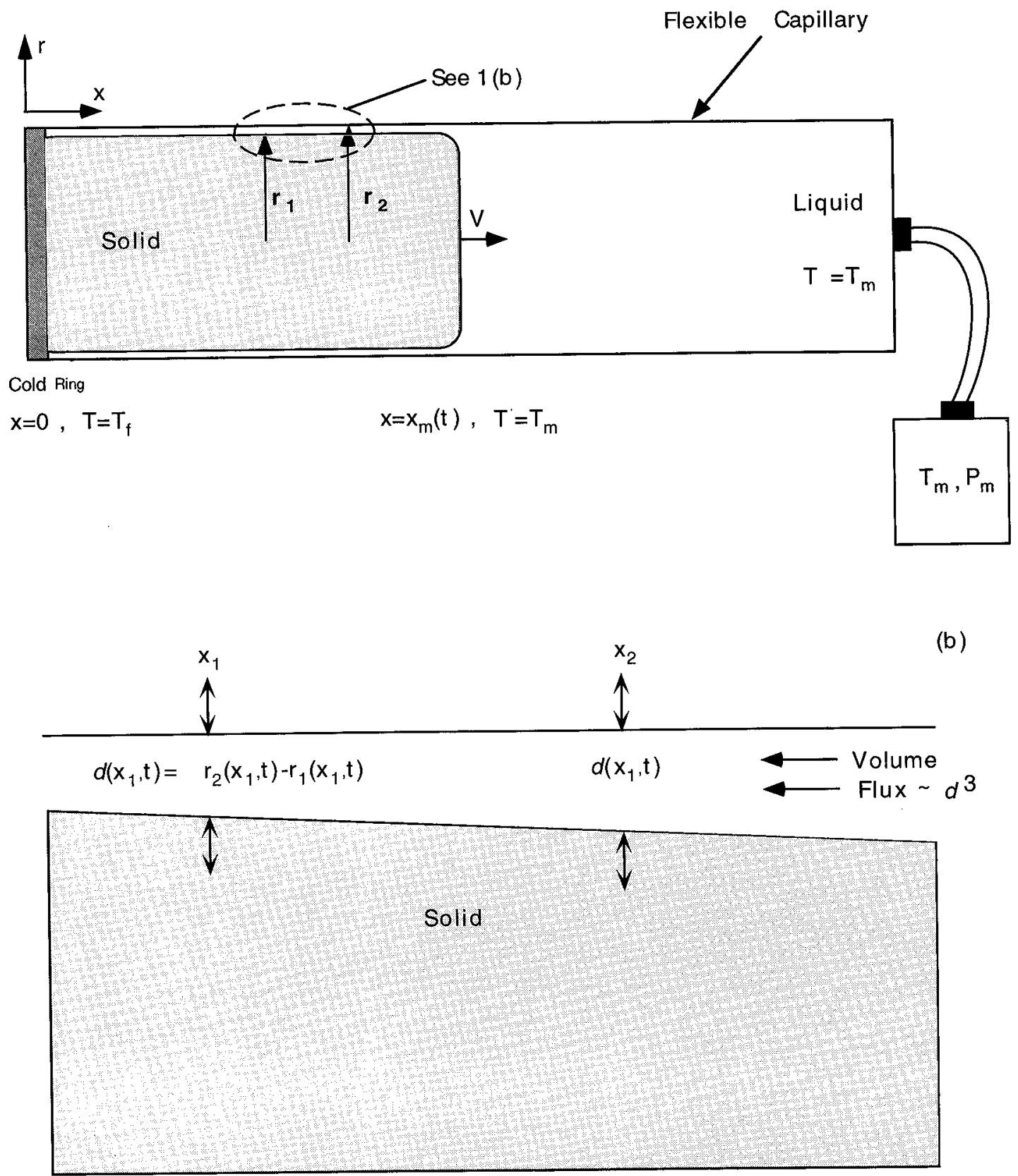

Figure 1. Schematic of the capillary tube gedanken experiment. (a) We take the cold ring at $x=0$ to be the plane of symmetry of a flexible capillary tube (which extends from $-\propto$ to $+\propto$ ) filled with the material of interest (ice/water in this case). With the exception of the small region adjacent to the ring, the walls of the tube are insulated so that only axial heat currents are allowed. Initially we envisage the system to consist of solid and liquid in coexistence at $T=T_{\mathrm{m}}$ so that we must have $T_{\mathrm{f}}=T_{\mathrm{m}}$. The liquid region is allowed to communicate with a reservoir which has independent temperature and pressure control. We begin an experiment by cooling the cold ring to $T_{\mathrm{f}}<T_{\mathrm{m}}$. This establishes a temperature gradient across the solid which drives it into the isothermal melt. The radius of the solid finger is $r_{1}$ and that of the tube is $r_{2}$. At a given temperature the interfacially melted film has thickness $r_{2}-r_{1}=d=\lambda_{v=3} t_{\mathrm{r}}{ }^{-1 / 3}$ if the dominant interactions are nonretarded van der Waals. The position of the moving "bulk" interface $x_{\mathrm{m}}(t)$ is unknown in the transient problem. (b) The imposed temperature gradient establishes a thermomolecular pressure gradient (see text) which drives a lubrication flow in the region between the solid and the membrane with a volume flux $\mathrm{Q} \propto d^{3}$. Material in excess of the thickness given by eq 2 solidifies, and the membrane is distorted in a manner to be determined.

capillary $k$. The deformation of the tube takes place on a time scale that is rapid compared to the variation in the temperature gradient. ${ }^{9}$ Hence, the thermomolecular pressure gradient is essentially uniform over the same time scale. Yet, since the premelted film is thickest near the high-temperature region of the nose and decays rapidly toward the cold ring, the maximum gradient in the flow occurs near the nose, thereby causing most of the heave in that region. At the lower temperatures, the film flow is weak and there is little further heave, resulting in an approximately uniform displacement of the capillary wall away from the nose. Since the volume flux driving the deformation decreases at a rate that scales with the rate of motion of the bulk interface $\left(\propto t^{-1 / 2}\right)$, the deformed region of the capillary maintains the same shape while the solid finger grows down the tube. The much larger displacement near the cold finger is a remnant from the initial moments when the temperature gradient and the thermomolecular pressure gradient were large throughout the then small domain $0<x<x_{\mathrm{m}}$.

In Figure $2 \mathrm{~b}$ we show the case where the temperature gradient is held at a constant value $\mathrm{G}=\left(T_{\mathrm{m}}-T\right) / x$, which removes one equation and reduces the analysis to the appropriate modification of eq 8 which possesses a different similarity solution. Here 

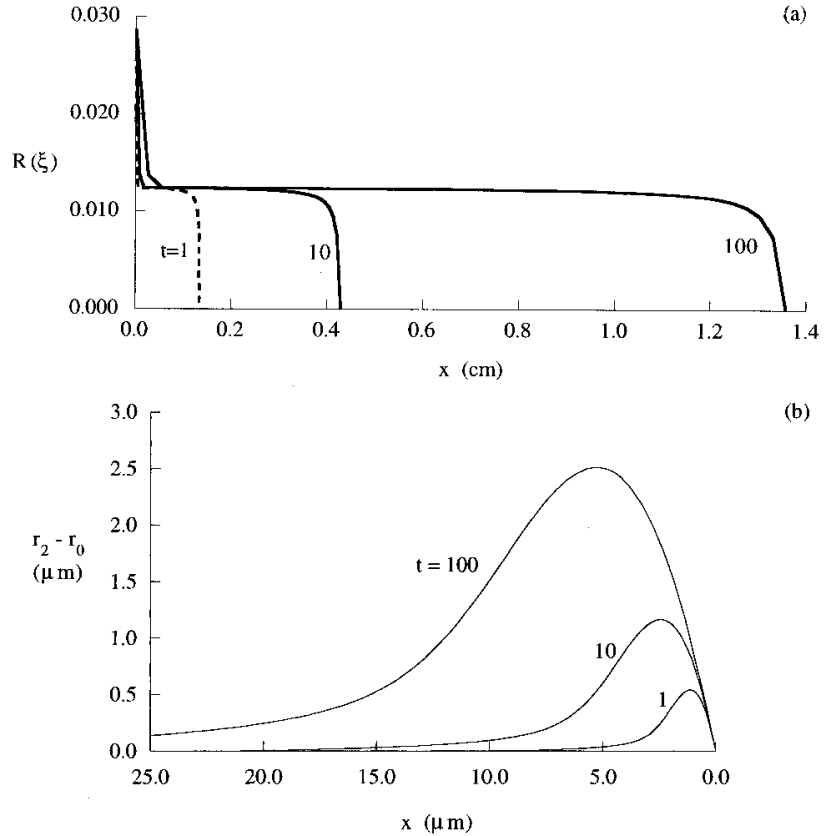

(b)

Figure 2. (a) Transient solution for the dimensionless wall displacement $R$ as a function of position at $t=1,10$, and $100 \mathrm{~min}$. The material parameters are for pure $\mathrm{H}_{2} \mathrm{O}$ ice, and $k=3 \times 10^{9} \mathrm{~Pa} \mathrm{~m}^{-1}$. (b) Dimensional deformation as a function of position at $t=1,10$, and $100 \mathrm{~h}$. We have chosen $\mathrm{G}=1 \mathrm{~K} \mathrm{~m}^{-1}$. Note the marked contrast with the solution of the transient problem.

the bulk solid-liquid interface is located at the fixed position $x=0$, and $x$ increases toward the cold ring. Note the striking contrast with the transient case. A constant $G$ drives a constant volume flux gradient, and thermomolecular pressure continually draws more fluid into the film where it freezes and deforms the capillary tube. The position of maximum deformation $x_{\max }$ moves toward the colder end of the tube as

$$
x_{\max } \approx 1.14\left(\frac{\pi k \lambda_{v}{ }^{3} T_{\mathrm{m}}}{12 \rho_{\mathrm{s}} n \mathrm{G}}\right)^{1 / 3} t^{1 / 3}
$$

and the maximum displacement $\left(r_{2}-r_{0}\right)_{\max }$ increases with time according to

$$
\left(r_{2}-r_{0}\right)_{\max } \approx 0.541\left(\frac{\lambda_{v}^{3}}{12 n}\right)^{1 / 3}\left(\frac{\rho_{\mathrm{s}} \mathrm{G}}{\pi k T_{\mathrm{m}}}\right)^{2 / 3} t^{1 / 3}
$$

In principal, large deformations can be achieved by increasing $\mathrm{G}$ and decreasing $k$. However, the migration of the peak toward lower temperatures will be most dramatic when $G$ is small and $k$ is large. Finally, we note that the width of the peak also scales as $(k / G)^{1 / 3} t^{1 / 3}$, so will spread for large values of $k / G$, and will narrow and shift toward the bulk solid-liquid interface as $k / G$ decreases. Detailed experimental tests of this geometry have yet to be conducted; however, the qualitative features of the constant temperature gradient case are consistent with the experimental findings of Wilen and Dash. ${ }^{10}$ Since the elasticity of the membrane in their experiment induces a curvature, there were nonlocal effects that had to be considered for quantitative comparisons. We describe these next.

Wilen and Dash ${ }^{10}$ observed premelting dynamics at ice single crystal interfaces formed against a flexible polymer membrane in an imposed constant temperature gradient $G$. Their cell has a radial configuration, but because the membrane height deformations are much less than the disk radius, it is treated in a one-dimensional slab geometry (Figure 3a). The height of

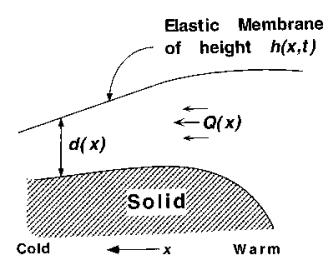

(a)

(b)

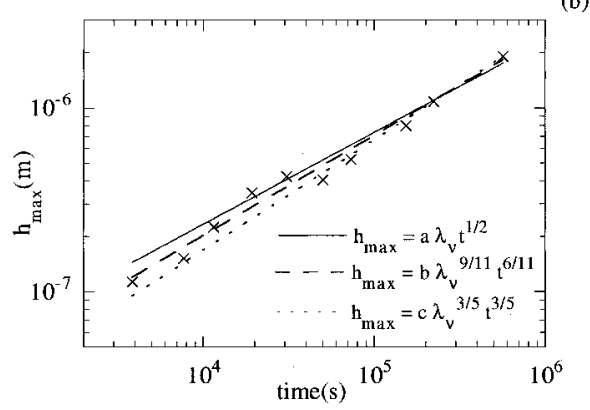

(c)

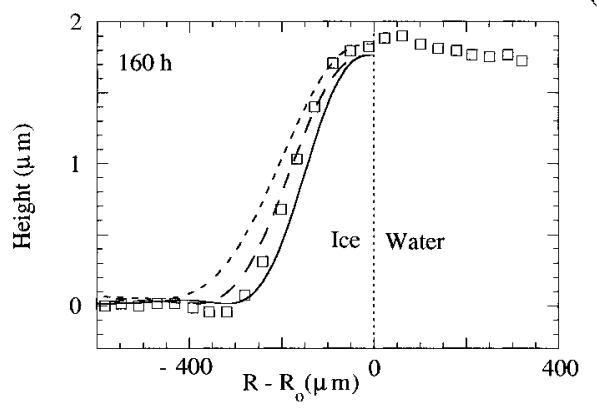

Figure 3. (a) Local configuration of a radial frost heave cell treated in a one-dimensional slab geometry. ${ }^{11}$ This differs from the capillary tube in that the membrane exerts a restoring force proportional to its curvature. The temperature gradient $\mathrm{G}$ is constant so $t_{\mathrm{r}}=\mathrm{G} x / T_{\mathrm{m}}$, and the film thickness $d$ depends only on $x$. The thermomolecular pressure gradient drives the evolution of the membrane height $h(x, t)$ relative to an initial reference height $h\left(x, t_{0}\right)$. (b) Fits of the maximum height of the membrane $h_{\max }(t)$ as given by eq 14 . The three power laws $t^{1 / 2}$, $t^{6 / 11}$, and $t^{3 / 5}$ arise from the short- and long-range electrostatic $(v=3 / 2$ and $v=2)$ and nonretarded van der Waals $(v=3)$ interactions, respectively. For each interaction we fit $\left((\nu-1) \sigma^{\nu-1} \Delta \gamma\right)^{-(9 /(4 v+3))}$, related to $\lambda_{v}$ as defined in eq 2. $a, b$, and $c$ are material constants, and $\lambda_{v=3 / 2}$ $=0.0337 \AA, \lambda_{v=2}=0.2101 \AA$, and $\lambda_{v=3}=1.3759 \AA$. (c) Comparison of the theoretical predictions and the experimental values described in ref 10 for the membrane height $h(x, t)$ at $160 \mathrm{~h} . R-R_{0}$ is the experimental $x$-coordinate. At the bulk ice/water interface, $R=R_{0}$ and $\mathrm{G}=0.92 \mathrm{~K} \mathrm{~cm}^{-1}$. The predictions for the short- and long-range electrostatic $(v=3 / 2$ and $v=2)$ and the nonretarded van der Waals $(v$ $=3$ ) interactions are shown by the solid, dashed, and dotted lines, respectively. For orientation "Ice" $\left(R-R_{\mathrm{o}}<0\right)$ and "Water" $\left(R-R_{0}\right.$ $>0$ ) refer to regions where the bulk phases are stable. For $R-R_{0}<$ 0 an interfacial water film coexists between the membrane and bulk ice.

the membrane, $h=h(x, t)$, measured relative to an initial reference height $h\left(x, t_{0}\right)$ is observed to change in response to the flow of premelted water in a narrow temperature range near $T_{m}$, with a relative lack of membrane deformation at lower temperatures. Initially, this was interpreted as an abrupt decrease to $d=0$, consistent with an interfacial free energy that decreases monotonically with film thickness at long range, but which possesses a local minimum at shorter range. Subsequently, using the analysis that follows, we found an alternative explanation.

In this geometry we find a family of mass conservation equations, each one corresponding to a different power law intermolecular interaction as 


$$
\partial_{t} h+\Lambda_{v} \partial_{x}\left[x^{-3 / v}\left(1+\alpha h_{x x x}\right)\right]=0
$$

where the term in the square brackets is the volume flux per unit breadth through the film of thickness given by eq 2 . Since $t_{\mathrm{r}}=\mathrm{G} x / T_{\mathrm{m}}$ and $d^{3} \propto x^{-3 / v}$, the first term in the square brackets displays the dynamical role of the interactions. The membrane possesses a tension, $\tilde{\sigma}$, so when it is distorted, it exerts a pressure on the solid proportional to the curvature. ${ }^{14}$ The gradient in this pressure is the $\alpha h_{x x x}$ term in the square brackets and displays the nonlocal effect of the membrane deformation. The coefficients are

$$
\alpha=\frac{\tilde{\sigma} T_{\mathrm{m}}}{\rho_{\mathrm{s}} q_{\mathrm{m}} \mathrm{G}} \quad \text { and } \quad \Lambda_{v}=\frac{\lambda_{v}{ }^{3} \rho_{\mathrm{l}} q_{\mathrm{m}}}{12 \mu}\left(\frac{T_{\mathrm{m}}}{\mathrm{G}}\right)^{(3-v) / v}
$$

Equation 12 possesses a similarity solution leading to a parameter free family of fourth-order, dimensionless ordinary differential equations that we solved numerically. ${ }^{11}$

We then performed an experimental test of the scaling prediction $^{11}$ for the time evolution of the maximum height $h_{\max }$ of the membrane:

$$
h_{\max }=\alpha^{-1}\left(\Lambda_{v} \alpha t\right)^{3 v /(4 v+3)} f_{\max }
$$

The coefficient depends only on material constants multiplied by $\left((v-1) \sigma^{\nu-1} \Delta \gamma\right)^{-(9 /(4 v+3))}$, which we fit to determine the slope of the interfacial potential I $(d)$. As discussed above, when $v$ $=3$, we are estimating the Hamaker constant. The fits span several decades in time (Figure $3 \mathrm{~b}$ ). The coefficient for a given interaction then allows us to predict $h(x, t)$ by solving the relevant evolution equation. The agreement between theory and experiment (Figure 3c) is best in the case of electrostatic interactions which always dominate van der Waals at long range and, depending on the surface charge density, may also dominate at short range. ${ }^{6}$ However, the observations cannot be accounted for by a single electrostatic interaction. Since the latter dominates at long range, we are confident in the calculation in the region of maximal heave. Superimposed other interactions might also play a role, and crossovers from one type to another can be treated with a strictly numerical approach. Our most important result lies in the new interpretation of the experiments consistent with a monotonically decreasing interfacial free energy over the entire range of film thicknesses. Although the low-temperature dynamics are slow, nonlocal effects ensure that they still play a role.

These treatments focus on the role of interfacial premelting in the transport of unfrozen water, but important effects such as curvature, confinement, interfacial roughness, disorder, and impurities have yet to be understood in this context. For example, confinement alone stabilizes liquid well below its bulk freezing point. ${ }^{15}$ With regard to the mathematical structure of these systems, similarity solutions appear often in lubrication flows ${ }^{16}$ but rarely receive experimental scrutiny over the requisite time scales. Therefore, we hope that our theories stimulate further work in premelting dynamics and related fields of wetting phenomena and thin film dynamics.

Acknowledgment. We acknowledge extremely useful conversations with M. P. Brenner, J. W. Cahn, J. G. Dash, S. C. Fain, J. F. Nye, R. Netz, M. Schick, and H. A. Stone. This work has been generously supported by Grants ONR N0001494-1-0120, ARO DAAH34-96-1-0433, and NSF OPP 95-23513 and the Natural Environment Research Council of the U.K.

\section{References and Notes}

(1) Dash, J. G. Films on Solid Surfaces; Academic: New York, 1974.

(2) Schick, M. In Liquids at Interfaces; Les Houches Session XLVIII; Charvolin, J., Joanny, J. F., Zinn-Justin, J., Dietrich, S. In Phase Transitions and Critical Phenomena; Domb, C., Lebowitz, J., Ed.s; Academic: New York, 1988; Vol. 12. Evans, R. J. Phys.: Condens. Matter 1990, 2, 8989

(3) Schick, M. Ber. Bunsen-Ges. Phys. Chem. 1996, 100, 272

(4) Julicher, F.; Lipowsky, R. Phys. Rev. E 1996, 53, 2670. 58,115

5) Dash, J. G.; Fu, H.-Y.; Wettlaufer, J. S. Rep. Prog. Phys. 1995

(6) Wilen, L. A.; Wettlaufer, J. S.; Elbaum. M.; Schick, M. Phys. Rev. $B$ 1995, 52, 12426 .

(7) Löwen, H. Phys. Rep. 1994, 237, 249.

(8) Netz, R.; Andelman, D. Phys. Rev. E 1997, 55, 687.

(9) Wettlaufer, J. S.; Worster, M. G. Phys. Rev. E 1995, 51, 4679.

(10) Wilen, L. A.; Dash, J. G. Phys. Rev. Lett. 1995, 74, 5076.

(11) Wettlaufer, J. S.; Worster, M. G.; Wilen, L. A.; Dash, J. G. Phys. Rev. Lett. 1996, 76, 3602.

(12) de Gennes, P. G. Rev. Mod. Phys. 1985, 57, 827. Leger, L.; Joanny, J. F. Rep. Prog. Phys. 1992, 55, 431. Israelachvili, J. N. Intermolecular and Surface Forces; Academic: New York, 1992.

(13) Gilpin, R. R. Water Resources Res. 1980, 16, 918.

(14) The membrane energy is dominated by surface tension, $\tilde{\sigma}=1 \mathrm{psi}$ $\mathrm{cm}^{-1}$, Young's modulus $E \approx 600 \mathrm{psi}$, and the membrane is $0.025 \mathrm{~mm}$ thick, so that the bending energy contributes only about $1 \%$ to the membrane energy.

(15) Christenson, H. K. Phys. Rev. Lett. 1995, 74, 4675.

(16) Bertozzi, A. L.; Brenner, M. P.; Dupont, T. F.; Kadanoff, L. P. In Trends and Perspectives in Applied Mathematics; Sirovich, L., Ed.; Applied Mathematical Sciences; Springer-Verlag: New York, 1994; Vol. 100, p 155. Almgren, R.; Bertozzi, A. L.; Brenner, M. P. Phys. Fluids 1996, 8, 1356. 